Publ. RIMS, Kyoto Univ.

19 (1983), 753-763

\title{
Convergence of Martingales on a Riemannian Manifold
}

By

\author{
Richard W. R. DARLING*
}

\begin{abstract}
When the scalar quadratic variation of a martingale on a Riemannian manifold is finite almost surely, then the martingale converges almost surely in the one-point compactification of the manifold. A partial converse due to Zheng Wei-an is also proved. No curvature conditions on the manifold are required.
\end{abstract}

\section{$\S 0 . \quad$ Introduction}

Let $M$ be an $n$-dimensional differential manifold with a Riemannian metric $g$, and let $\Gamma$ be a metric connection for $M$. We consider a stochastic process $X$ on $M$ with continuous trajectories, and such that the image of $X$ under every $C^{2}$ function is a real-valued semimartingale. For a moment think of $M$ as an oblong ball resting on the plane $E$, with $X_{0}$ being the point of contact. A well-known procedure in stochastic differential geometry is the so-called stochastic development, in which, by means of the metric connection $\Gamma$, the manifold is rolled along the plane $E$ without slipping, such that $X_{t}$ is the point of contact at each time $t$. If we imagine the path of $X$ as being traced in ink, a 'developed' process $Z$ is printed onto $E$.

The process $X$ is called a $\Gamma$-martingale if $Z$ is a local martingale on $E$. The purpose of this paper is to establish two results about $\Gamma$-martingales: first, that on the set where the scalar quadratic variation of $Z$ is finite the process $\left(X_{t}\right)$ tends almost surely to a limit $X_{\infty}$ in the one-point compactification of $M$; secondly, that on the set where $X_{\infty}$ exists and lies in $M$ itself, the scalar quadratic variation of $Z$ is finite. The first

Communicated by S. Matsuura, October 13, 1982.

* Department of Mathematics, University of Southern California, Los Angeles, CA 90089, U.S.A. 
result is due to the author [3], the second to Zheng [8]. The remarkable aspect of these results is that no curvature assumptions are made about the manifold; $M$ does not even have to be complete.

The proofs of these results make use of stochastic calculus on principal fibre bundles (Darling [3]). The first proof exploits the Ito formula for the image of $X$ under $C^{\infty}$ functions $f$ on $M$ with compact support. The second proof uses an immersion argument and a subtle lemma about continuous semimartingales.

\section{$\S$ H. Basic Stochastic Notations}

When $Y$ is a real-valued stochastic process, then $Y_{\infty}^{*}$ denotes $\sup _{t}\left|Y_{t}\right|$; if $T$ is a stopping-time, then $Y^{T}$ is the process $Y_{t}^{T}=Y_{t \wedge T}$. If $Y$ is a continuous semimartingale with a local martingale part $N$ in the standard decomposition, then the scalar quadratic variation process of $Y$ is written $\langle Y, Y\rangle$; of course $\langle Y, Y\rangle_{t}=\langle N, N\rangle_{t}$. When $Y$ is $\mathbb{R}^{n}$-valued with components $\left(Y_{t}^{1}, \cdots, Y_{t}^{n}\right)$, then $\langle Y, Y\rangle_{t}$ denotes $\sum_{i}\left\langle Y^{i}, Y^{i}\right\rangle_{t}$. The matrixvalued differential $\left(\left(d\left\langle Y^{i}, Y^{j}\right\rangle_{t}\right)\right)$ will be abbreviated to $(d Y \otimes d Y)_{t}$.

A Stratonovitch differential is denoted by $\circ$, as in $L_{t} \circ d Y_{t}$. However ' $\circ$ ' is also used to denote composition of mappings, as in $\varphi \circ X$.

Suppose $X$ is a process with values in a differential manifold $M$, and $(W, \varphi)$ is a chart for $M$. If $K$ is a real-valued bounded predictable process which vanishes outside the random set $\{X \in W\}$, and if $\varphi \circ X$ is a semimartingale in $R^{n}$, then it makes sense to refer to

$$
\int_{0}^{t} K_{s} d\left(\varphi\left(X_{s}\right)\right), \text { denoted } \int_{0}^{t} K_{s} d X_{s}^{\varphi}
$$

and

$$
\int_{0}^{t} K_{s}(d(\varphi \circ X) \otimes d(\varphi \circ X))_{s}, \text { denoted } \int_{0}^{t} K_{s}(d X \otimes d X)_{s}^{\varphi}
$$

The last expression could also be described as the $n \times n$ matrix with $(i, j)^{t h}$ entry

$$
\int_{0}^{t} K_{s} d\left\langle X^{i}, X^{j}\right\rangle_{s}
$$

where $X^{i}=i^{t h}$ co-ordinate process of $\varphi \circ X$. 


\section{$\S 2$. Constructions and Formulae from Stochastic Differential Geometry}

A good reference for this section is Meyer [4], but the notation is more in the spirit of Darling [3].

1. Let $M$ be a smooth $n$-dimensional manifold; for brevity, we shall often denote the model space by $\mathbb{E}$ instead of $\mathbb{R}^{n}$. A semimartingale on $M$ will mean a process $X$ with almost surely continuous paths on $M$, whose image under every $C^{2}$ function from $M$ to $\mathbb{R}$ is a real-valued semimartingale. (The definition is due to Schwartz [6].)

2. Let $\eta$ be a first-order differential form (' 1 -form') on $M$. If $(W, \varphi)$ is a chart for $M$, then $\eta$ has the local representation $(\varphi(x), a(x))$ $\in \mathbb{E} \times \mathbb{E}^{*}$ at each $x$ in $W$, where $\eta=\varphi^{*} a$ on $W$ (meaning that $\eta(x)$ (v) $=a(x)\left(T_{x} \varphi(v)\right)$ for $v$ in $\left.T_{x} M\right)$. On the random set $\{X \in W\}$, the following differential makes sense and is intrinsic (for the notation, see Section 1):

$$
a\left(X_{t}\right) d X_{t}^{\varphi}+\frac{1}{2} D a\left(X_{t}\right)(d X \otimes d X)_{t}^{\varphi}
$$

Consequently there is a unique real-valued process $Y$ with $Y_{0}=0$ such that $d Y_{t}$ equals the last expression on $\{X \in W\}$, for each chart $(W, \varphi)$. The process $Y$ is called the Stratonovitch integral of the 1 -form $\eta$ along the semimartingale $X$, and we usually write

$$
Y=(S) \int_{X} \eta, Y_{t}=(S) \int_{X_{0}^{t}} \eta
$$

(Meyer would omit the symbol $(S)$.)

3. Let $p: P \rightarrow M$ be a principal fibre bundle with group $G$, which is a sub-bundle of the bundle of linear frames. Hence for each $x$ in $M$ and each $u$ in $p^{-1}(x), u$ is a linear isomorphism from $\mathbb{E}$ into $T_{x} M$. Let $\omega$ be a connection 1-form on $P$. Various authors have shown (Meyer [4, p. 80], Darling [3, pp. 30-34], Shigekawa [7]) that given a semimartingale on $M$ and an initial frame $U_{0}$ in $p^{-1}\left(X_{0}\right)$, there is a unique 
semimartingale $U$ on $P$, called the horizontal lift of $X$ to $P$ through $\omega$, satisfying the equations:

$$
(S) \int_{U} \omega=0, p\left(U_{t}\right)=X_{t}
$$

For a given connection 1 -form $\omega$ and a given initial frame $U_{0}$, the stochastic development of $X$ into $\boldsymbol{E}$ is the $\boldsymbol{E}$-valued semimartingale $Z$ defined by:

$$
Z=(S) \int_{U} \theta
$$

where $\theta$ is the canonical 1 -form on $P$, namely the $\boldsymbol{E}$-valued 1 -form defined by:

$$
\theta(u)(\xi)=u^{-1}\left(T_{u} p(\xi)\right), \quad u \in P, \xi \in T_{u} P
$$

The usefulness of the horizontal lift and the stochastic development is that they allow formulas related to the process $X$ on $M$ to be written down in 'absolute' terms, without reference to any system of co-ordinates on $M$. For example, let $f: M \rightarrow \mathbb{R}$ be a smooth function. Then $d f\left(X_{s}\right)$ $\in T_{X_{s}}^{*} M$. Let $\left(e_{1}, \cdots, e_{n}\right)$ be an orthonormal basis for $E$, and write $Z_{t}=\left(Z_{t}^{1}, \cdots, Z_{t}^{n}\right)$ with respect to this basis. Then $U_{s}\left(e_{i}\right)$ is a tangent vector at $X_{s}$ for each $i$, and so $d f\left(X_{s}\right)\left(U_{s}\left(e_{i}\right)\right)$ is real-valued. Likewise $\nabla d f\left(X_{s}\right)\left(U_{s}\left(e_{i}\right), U_{s}\left(e_{j}\right)\right)$ is real-valued, where $\nabla$ is the covariant derivative induced by $\omega$ (assuming $P$ is the linear or the orthonormal frame bundle). Versions of the following 'Ito formula' have been given by many authors (e.g. Meyer [4], Bismut [1]) but we give the version appearing in Darling [3, p. 24];

$$
\begin{aligned}
f\left(X_{t}\right)-f\left(X_{0}\right)= & \int_{0}^{t}\left(d f\left(X_{s}\right) \circ U_{s}\left(e_{i}\right)\right) d Z_{s}^{i} \\
& +\frac{1}{2} \int_{0}^{t} \nabla d f\left(X_{s}\right)\left(U_{s}\left(e_{i}\right), U_{s}\left(e_{j}\right)\right) d\left\langle Z^{i}, Z^{j}\right\rangle_{s} .
\end{aligned}
$$

In fact the use of a basis for $\mathbb{E}$ is not necessary, and we may abbreviate to:

$$
\begin{aligned}
f\left(X_{t}\right)-f\left(X_{0}\right)= & \int_{0}^{t}\left(d f\left(X_{s}\right) \circ U_{s}\right) d Z_{s} \\
& +\frac{1}{2} \int_{0}^{t} \nabla d f\left(X_{s}\right)(U d Z \otimes U d Z)_{s}
\end{aligned}
$$


4. Suppose $(M, g)$ is a Riemannian manifold and $P$ is the bundle of orthonormal frames. For the sake of easy comparison with Meyer $\left[4\right.$, p. 64], we shall work in local co-ordinates $\left(x^{i}\right)$. Take an orthonormal basis $\left(e_{1}, \cdots, e_{n}\right)$ for $\boldsymbol{E}$ and write $U_{s}\left(e_{k}\right)$ as $U_{k}^{i}(s) D_{i}$ in the tangent space to $M$ at $X_{s}$. Then equation (5) implies

$$
d X_{s}^{i}=U_{k}^{i}(s) d Z_{s}^{k}+\{\text { terms of bounded variation }\}
$$

Hence

$$
\begin{aligned}
g_{i j}\left(X_{s}\right) d\left\langle X^{i}, X^{j}\right\rangle_{s} & =g_{i j}\left(X_{s}\right) U_{k}^{i}(s) U_{m}^{j}(s) d\left\langle Z^{k}, Z^{m}\right\rangle_{s} \\
& =\sum_{m} d\left\langle Z^{m}, Z^{m}\right\rangle_{s}
\end{aligned}
$$

by the orthonormality of the frames $U_{s}$. It follows that

$$
g_{i j}\left(X_{s}\right) d\left\langle X^{i}, X^{j}\right\rangle_{s}=d\langle Z, Z\rangle_{s}
$$

and we can define the scalar quadratic variation of $X$ (with respect to the metric $g$ ) by: $\langle X, X\rangle_{t}=\langle Z, Z\rangle_{t}$.

5. A semimartingale $X$ on $M$ is said to be a $\Gamma$-martingale if $Z$ is an ( $\boldsymbol{R}^{n}$-valued) local martingale, where $\Gamma$ refers to the linear connection for $M$ induced by the connection 1-form $\omega$ on the bundle of linear or orthonormal frames. In the case where $(M, g)$ is Riemannian, $P$ is the orthonormal frame bundle, (and so $\Gamma$ is a metric connection) $X$ is called a square-integrable $\Gamma$-martingale if $Z$ is a square-integrable martingale, which implies that $\langle Z, Z\rangle_{\infty}<\infty$. More information about $\Gamma$-martingales may be found in Darling [2], [3].

\section{§ 3. Convergence Theorem for Martingales on a Riemanniam Manifold}

Theorem A. We assume that $(M, g)$ is a Riemannian manifold with a metric connection $\Gamma$ (possibly with torsion), and $X$ is a $\Gamma$. martingale on $M$. Then the limit $X_{\infty}=\lim _{t} X_{t}$ exists almost surely in the one-point compactification (=Alexandroff compactification) $M \cup\{\delta\}$ of $M$, on the set where $\langle X, X\rangle_{\infty}$ is finite.

Theorem B. (Zheng [8]). The assumption are the same as for 
Theorem $A$. On the set where $X_{\infty}$ exists and lies in $M$, we have: $\langle X, X\rangle_{\infty}<\infty$ almost surely.

Remark. Let $(M, g)$ be a Riemannian manifold on which the Brownian motion $B$ has a finite lifetime almost surely. In [5], Meyer shows how to construct a time-change $t \rightarrow \tau_{t}$ such that if $X_{t}=B_{\tau_{t}}$, then $X$ is a square-integrable martingale (with respect to the Levi-Civita connection), and $X_{\infty}$ is the point at infinity. So the compactification of $M$ really is necessary for the theorem.

The proof of (B) comes in Section 5. The proof of (A) is preceded by a pair of easy lemmas, both of which were noticed by P. A. Meyer.

Lemma 1. Let $E$ be a finite-dimensional inner product space, with dual $E^{*}$. Let $c$ be a bilinear form on $E$ and $q$ a positive semidefinite symmetric bilinear form on $E^{*}$. Then with respect to any orthonormal basis for $E$,

$$
\left|c_{i j} q^{i j}\right| \leqq \sum_{i} q^{i i}\|c\|
$$

where

$$
\|c\|=\sup \left\{c(a, a): \sum_{i}\left(a^{i}\right)^{2}=1\right\} .
$$

Furthermore if $c$ is also positive semidefinite, then

$$
c_{i j} q^{i j} \geqq \sum_{i} q^{i i} \alpha(c)
$$

where $\alpha(c)=\inf \left\{c(a, a): \sum_{i}\left(a^{i}\right)^{2}=1\right\}$.

Proof. Both sides of (8) are independent of the choice of basis. Take a basis so that $q$ has a diagonal matrix. Then

$$
\begin{aligned}
\left|c_{i j} q^{i j}\right| & =\left|\sum_{i} c_{i i} q^{i i}\right| \leqq\left(\sum_{i} q^{i i}\right) \sup _{i}\left|c_{i i}\right| \\
& =\left(\sum_{i} q^{i i}\right) \sup _{i}\left|c\left(e_{i}, e_{i}\right)\right|
\end{aligned}
$$

where the $e_{i}$ are the basis vectors. The proof $\left(8^{\prime}\right)$ is similar.

In the next lemma, $C_{K}^{\infty}(M)$ denotes the $C^{\infty}$ functions from $M$ to $\boldsymbol{R}$ 
with compact support.

Lemma 2. Let $C=\left\{\omega: \lim _{t \rightarrow \infty} f\left(X_{t}(\omega)\right)\right.$ exists for all $\left.f \in C_{K}^{\infty}(M)\right\}$. Then for all $\omega \in C, \lim _{t \rightarrow \infty} X_{t}(\omega)$ exists in $M \cup\{\delta\}$.

Proof. Suppose $\omega \in C$. If for some $f \in C_{K}^{\infty}(M), \lim _{t \rightarrow \infty} f\left(X_{t}(\omega)\right) \neq 0$, then $X_{t}(\omega)$ lies in some compact set $K$ for all sufficiently large $t$. Since $\lim _{t \rightarrow \infty} f\left(X_{t}(\omega)\right)$ exists for all $C^{\infty} f$ with support in $K$, it follows $\lim _{t \rightarrow \infty} X_{t}(\omega)$ exists in $K$. On the other hand if $\lim _{t \rightarrow \infty} f\left(X_{t}(\omega)\right)=0$ for all $f$, then for every compact set $K, X_{t}(\omega)$ lies outside $K$ for all sufficiently large $t$. Therefore $\lim _{t \rightarrow \infty} X_{t}(\omega)$ is the point at infinity.

Proof of Theorem A. As P. A. Meyer has pointed out, it suffices to treat the case where $\langle X, X\rangle_{\infty}$ is integrable. "For this case gives us the convergence of the stopped martingale $X^{T_{n}}$, where $T_{n}$ is the stoppingtime $\left.\inf \left\{t:\langle X, X\rangle_{t}\right\rangle n\right\}$; on the other hand, on the set $\left\{\langle X, X\rangle_{\infty}<\infty\right\}$ we have $T_{n}=+\infty$ for $n$ sufficiently large." We suppose henceforward that $\langle X, X\rangle_{\infty}$ is integrable. By the definition of $\langle X, X\rangle_{t}$ above, this says that $\langle Z, Z\rangle_{\infty}$ is integrable.

Let $f \in C_{K}^{\infty}(M)$. By formula (5) we may write

$$
f\left(X_{t}\right)-f\left(X_{0}\right)=\int_{0}^{t} a_{i}(s) d Z_{s}^{i}+\int_{0}^{t} b_{i j}(s) d\left\langle Z^{i}, Z^{j}\right\rangle_{s},
$$

where

$$
a_{i}(s)=d f\left(X_{s}\right) \circ U_{s}\left(e_{i}\right)
$$

and

$$
b_{i j}(s)=\frac{1}{2} \nabla d f\left(X_{s}\right)\left(U_{s}\left(e_{i}\right), U_{s}\left(e_{j}\right)\right)
$$

Let $Q_{t}$ denote the first integral on the right (a local martingale) and $A_{t}$ the second. Since $d f$ and $\nabla d f$ are bounded, and $U_{s}\left(e_{i}\right)$ is always of unit Riemannian length, there is a constant $K$ such that

$$
\sum_{i} a_{i}(s)^{2}<K, \quad \text { all } s
$$

and 


$$
\sup _{i}\left|b_{i i}(s)\right|<K, \quad \text { all } s \text {. }
$$

We shall now show that $Q$ is a square integrable martingale; for apply Lemma 1 with $c_{i j}=a_{i}(s) a_{j}(s), q^{i j}=d\left\langle Z^{i}, Z^{j}\right\rangle_{s}$, to deduce:

$$
\begin{aligned}
d\langle Q, Q\rangle_{s} & =a_{i}(s) a_{j}(s) d\left\langle Z^{i}, Z^{j}\right\rangle_{s} \\
& \leqq \sup \left\{\left|c_{i j} v^{i} v^{j}\right|: \sum_{i}\left(v^{i}\right)^{2}=1\right\} \sum_{i} d\left\langle Z^{i}, Z^{j}\right\rangle_{s} \\
& =\sup \left\{\left(a_{i}(s) v^{i}\right)^{2}: \sum_{i}\left(v^{i}\right)^{2}=1\right\} d\langle Z, Z\rangle_{s} \\
& \leqq K^{2} d\langle Z, Z\rangle_{s},
\end{aligned}
$$

using the Schwartz inequality. The convergence theorem for real-valued square-integrable martingales implies that $Q_{\infty}=\lim _{t \rightarrow \infty} Q_{t}$ exists a.s.. As for the process $A$, apply Lemma 1 again with $c_{i j}=b_{i j}(s), q^{i j}=d\left\langle Z^{i}, Z^{j}\right\rangle_{s}$ to deduce that

$$
\left|d A_{t}\right| \leqq K \sum_{i} d\left\langle Z^{i}, Z^{i}\right\rangle_{t}=K d\langle Z, Z\rangle_{t}
$$

Hence $A_{\infty}=\lim _{t \rightarrow \infty} A_{t}$ exists a.s.. By Lemma 2, this completes the proof.

\section{$\S 4$. A Result on Semimartingales in $\mathbb{R}^{n}$}

W. A. Zheng has proved the following result [8]:

Lemma 3. Let $Y$ be a real-valued continuous semimartingale, with canonical decomposition $Y=Y_{0}+N+A$. We suppose that $d A_{t}$ $\ll d\langle Y, Y\rangle_{t}=d\langle N, N\rangle_{t}$, so that one can write

$$
Y_{t}=Y_{0}+N_{t}+\int_{0}^{t} H_{s} d\langle N, N\rangle_{s}
$$

Then on the set

$$
C=\left\{Y_{\infty} \text { exists and is finite, and ess } \sup _{t}\left|H_{t}\right|<\infty\right\}
$$

the limit $\langle N, N\rangle_{\infty}$ is finite. (The ess $\sup _{t}$ is taken with respect to the measure $\left.d\langle N, N\rangle_{t}(\omega)\right)$.

We would like to apply the lemma in a more complicated situation. Suppose $Z=\left(Z^{1}, \cdots, Z^{q}\right)$ is a continuous martingale in $\boldsymbol{R}^{q}$, and suppose 
$Y$ is an $\boldsymbol{R}^{n}$-valued continuous semimartingale of the form

$$
Y_{t}=Y_{0}+N_{t}+A_{t}
$$

where

$$
N_{t}^{i}=\int_{0}^{t} J_{j}^{i}(s) d Z_{s}^{j}
$$

and

$$
A_{t}^{i}=\int_{0}^{t} H_{j k}^{i}(s) d\left\langle Z^{j}, Z^{k}\right\rangle_{s}
$$

Set $\left|H_{t}\right|=\sup _{i} \sup _{|x|<1}\left|H_{j k}^{i}(t) x^{j} x^{k}\right|$ and $H_{\infty}^{*}=$ ess $\sup _{t}\left|H_{t}\right|$. (The ess sup may be taken with respect to the measure $\left.d\langle Z, Z\rangle_{t}\right)$. Regarding $J(s)$ as a linear transformation from $\boldsymbol{R}^{q}$ to $\boldsymbol{R}^{n}$, define a random variable

$$
\gamma(J)=\inf _{t} \inf \left\{e^{T} J^{T}(t) J(t) e: e \in \mathbb{R}^{q},|e|=1\right\}
$$

Notice that in terms of $\alpha(\cdot)$ defined in Lemma 1 above,

$$
\gamma(J)=\inf _{t} \alpha\left(J^{T}(t) J(t)\right)
$$

Lemma 4. Let $\varepsilon>0$, and let the situation be as just described. On the set

$$
C=\left\{Y_{\infty} \text { exists and is finite, } H_{\infty}^{*}<\infty \text {, and } \gamma(J)>\varepsilon\right\}
$$

we have: $\langle Z, Z\rangle_{\infty}<\infty$ a.s..

Proof. A straightforward extension of Lemma 3 to the vector case shows that $\langle N, N\rangle_{\infty}<\infty$ a.s. on $C$. Apply Lemma 1, second part, with $c_{i j}=\sum_{k} J_{i}^{k}(t) J_{j}^{k}(t)$ and $q^{i j}=d\left\langle Z^{i}, Z^{j}\right\rangle_{t}$. We obtain:

$$
\begin{aligned}
d\langle N, N\rangle_{t} & =\left(\sum_{k} J_{i}^{k}(t) J_{j}^{k}(t)\right) d\left\langle Z^{i}, Z^{j}\right\rangle_{t} \\
& \geqq \alpha\left(J^{T}(t) J(t)\right) d\langle Z, Z\rangle_{t} \\
& \geqq \gamma(J) d\langle Z, Z\rangle_{t} \geqq \varepsilon d\langle Z, Z\rangle_{t}
\end{aligned}
$$

This completes the proof.

\section{§5. Proof of Theorem $\mathbb{B}$}

Let $\omega$ be the connection 1-form on the orthonormal frame bundle 
$O(M)$, associated with the metric connection $\Gamma$ for $(M, g)$. Given a $\Gamma$-martingale $X$ on $M$, take an initial frame at $X_{0}$, and form the corresponding horizontal lift $U$ of $X$ to $O(M)$ through $\omega$, and the stochastic development $Z$ of $X$ into $\mathbb{R}^{n}$, as in Section 2, part 3. By definition of $\Gamma$-martingale, $Z$ is a local martingale.

There exists an integer $p$ and an immersion $h$ of $M$ into $\mathbb{R}^{n+p}$. Let $Y$ denote the image of $X$ in $\mathbb{R}^{n+p}$ under $h$. It follows from the Ito formula (6) that

$$
Y_{t}=Y_{0}+\int_{0}^{t} J(s) d Z_{s}+\int_{0}^{t} H(s)(d Z \otimes d Z)_{s},
$$

where

$$
J(s)=\left(d h\left(X_{s}\right) \circ U_{s}\right) \in L\left(\boldsymbol{R}^{n} ; \boldsymbol{R}^{n+p}\right)
$$

and

$$
H(s)=\frac{1}{2} \nabla d h\left(X_{s}\right)\left(U_{s}(\cdot), U_{s}(\cdot)\right) \in L\left(\boldsymbol{R}^{n}, \boldsymbol{R}^{n} ; \boldsymbol{R}^{n+p}\right) .
$$

Since $h$ is an immersion, $d h(x): T_{x} M \rightarrow \mathbb{R}^{n+p}$ is $1-1$ at each $x$ in $M$, and is a smooth function of $x$. Consequently on each compact subset $K$ of $M$, we can find a $c$ greater than zero such that

$$
|d h(x)(v)|>c \text {, all } x \in K \text {, all unit vectors } v \text { in } T_{x} M \text {. }
$$

Since $U_{s}$ is an isometry from $\boldsymbol{R}^{n}$ to $T_{X_{s}} M$ for each $s$, it follows that on the set $Q(K):=\left\{X_{t}\right.$ lies in $K$ for all $\left.t\right\}$,

(i) $\inf _{t} \inf \left\{|J(t, \omega) e|: e \in \mathbb{R}^{n},|e|=1\right\}>c$

or in the notation of Lemma $4, r(J)>c$ on $Q(K)$. Moreover it is easy to see that

(ii) $H_{\infty}^{*}<\infty$ on $Q(K)$, in the sense of Lemma 4 .

(A minor technical point: if $H$ is not symmetric, replace $H_{j k}^{i}(s)$ by $\frac{1}{2}\left(H_{k j}^{i}(s)+H_{j k}^{i}(s)\right)$; this does not affect $\left.Y\right)$.

Let $C$ be the set $\left\{X_{\infty}\right.$ exists in $\left.M\right\}=\left\{Y_{\infty}\right.$ exists in $\left.F\right\}$. From Lemma 4 , it follows that on $C \cap Q(K),\langle Z, Z\rangle_{\infty}<\infty$ a.s.. But for every $\omega$ in $C$, there is some compact $K$ containing the whole trajectory $X .(\omega)$. Hence $\langle Z, Z\rangle_{\infty}<\infty$ a.s. on $C$, as desired. 


\section{Acknowledgements}

The author wishes to express his gratitude to his supervisor K. D. Elworthy and to P. A. Meyer for their help on a number of points. The correspondence of P. A. Meyer has been a great inspiration.

An early draft of this paper was prepared with the assistance of W. A. Zheng, who helped to correct Lemma 4. Some of the results of this paper will also appear in an article by P. A. Meyer in Séminaire de Probabilites XVII.

\section{References}

[1] Bismut, J. M., Principes de mecanique aleatoire, Springer Lecture Notes in Math., 866 (1981).

[2] Darling, R. W. R., Martingales in manifolds-definition, examples, and behaviour under maps, Springer Lecture Notes in Math., 921 (1982), 217-236.

[3] - Martingales on manifolds and geometric Ito calculus, $\mathrm{Ph}$. D. Thesis, University of Warwick, 1982 (copies available from the author).

[4] Meyer, P. A., Geometrie stochastique sans larmes, Springer Lecture Notes in Mathematics, 850 (1981), 44-102.

[5] - Le théorème de convergence des martingales dans les varietes Riemanniennes, Sem. Probabilites XVII, Lecture Notes in Mathematics, to appear (1983).

[6] Schwartz, L., Semi-martingales sur des varietes, et martingales conformes, Springer Lecture Notes in Math., 780 (1980).

[7] Shigekawa, Ichiro., On stochastic horizontal lifts, Z. f. W., 59 (1982), 211-221.

[8] Zheng, W. A., Sur le theoreme de convergence des martingales dans une variete Riemanienne, Sem. Probabilites XVII, Lecture Notes in Mathematics, to appear (1983). 
